\title{
Mineralische Rohstoffe und Nachhaltigkeit
}

\author{
Susanne Feiel, Peter Moser, Michael Tost und Anna Meyer \\ Resources Innovation Center Leoben, Montanuniversität Leoben, Leoben, Österreich \\ Eingegangen 4. Februar 2019; angenommen 5. Februar 2019; online publiziert 20. Februar 2019
}

Zusammenfassung: Mineralische Rohstoffe und nachhaltige Entwicklung sind sehr eng miteinander verbunden, da sie im gegenwärtigen Wirtschaftssystem die Basis der gesellschaftlichen Entwicklung bilden. Jedoch haben noch nie zuvor in der menschlichen Entwicklungsgeschichte von Menschen getriebene Aktivitäten einen so starken Einfluss auf die Biosphäre gehabt, so dass die Systemgrenzen des Planeten erreicht bzw. in manchen Bereichen bereits überschritten sind. Aus diesem Grund besteht eine der zentralen Herausforderungen darin, auch zukünftig leistbare Rohstoffe bereitzustellen, diese jedoch bei Reduktion ökologischer Auswirkungen zu produzieren. Nur so wird es gelingen, die von den Vereinten Nationen am 25. September 2015 verabschiedeten Nachhaltigkeitsziele (Sustainable Development Goals, SDGs), deren Intention es ist, Wohlstand für alle herbeizuführen, zu erreichen. Eine Veränderung der momentanen neo-klassischen Industrie- und Wirtschaftsmodelle ist dafür dringend notwendig, denn diese wurden zu einem Zeitpunkt entwickelt, zu dem es auf der Welt noch viel weniger Menschen gab, einer sogenannten "leeren Welt", in der sich die verwendeten Ressourcen in Relation zu der Weltbevölkerung leicht wieder erholten und somit der vom Menschen erzeugte Einfluss von der Natur im Verhältnis noch absorbierbar war.

Schlüsselwörter: Nachhaltigkeit, Rohstoffsektor, Sustainable Development Goals, Verantwortlicher Konsum und Produktion, Planetary Boundaries, Anthropozän

\section{Raw Materials and Sustainability}

Abstract: Mineral raw materials and sustainable development are directly connected, as they form the basis for the societal development in the current economic system. Never before in human development, however, men

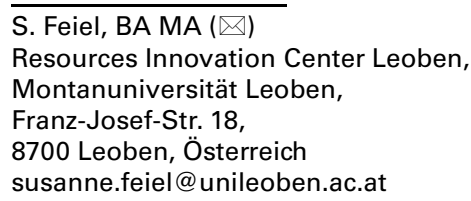

driven activities have had such an enormous impact on the biosphere so that the system boundaries of the planet have been reached or partly even crossed. For this reason, the main challenge of supplying affordable raw materials in the future is the reduction of the ecological impact of mineral production on the environment. Only in this way the sustainable development goals adopted by the United Nations on September 25th 2015, that aim at increasing global wealth for everybody, can be successfully realized. A change of the current neo-classical industrial and economic models seems to be urgent as they were developed and implemented in an "empty world" with a significantly lower world population than today, at a time when humancreated impact on the environment could still be absorbed by nature.

Keywords: Sustainability, Raw Materials Sector, Sustainable Development Goals, Responsible Consumption and Production, Planetary Boundaries, Anthropocene

\section{Herausforderungen}

Mit der durch den Menschen getriebenen, weiter wachsenden ökonomischen Leistung aller Länder und mit der weiter wachsenden Weltbevölkerung, die sich erst im Jahre 2100 bei rund $11 \mathrm{Mrd}$. stabilisieren soll [1], ist eine Verdoppelung des Rohstoffbedarfs bis 2050 [2] prognostiziert. Rohstoffe bilden im gegenwärtigen System die Basis der menschlichen Entwicklung, seien es Metalle, Bau-, Industrie-, Energie- oder Biorohstoffe. Noch nie zuvor haben von Menschen getriebene Aktivitäten einen so starken Einfluss auf die Biosphäre gehabt [3], und die erwähnten Faktoren ökonomische Leistung und Bevölkerungswachstum sind auch laut dem United Nations Environmental Programme - UNEP - ein omnipräsenter Treiber der Veränderung der Umwelt, vor allem die Aspekte Energie, Transport, Urbanisation und Globalisierung betreffend. Der Eingriff in die Natur durch den Menschen ist so groß, dass man unsere Gegenwart sogar als ein neues Zeitalter, nämlich als Anthropozän [4] bezeichnen kann. Durch Umsetzung der von den 
Vereinten Nationen am 25. September 2015 verabschiedeten Nachhaltigkeitsziele (Sustainable Development Goals, SDGs), die Wohlstand für alle bei gleichzeitiger Reduktion der Auswirkungen ökonomischer Aktivitäten fordern, wird eine weitere Erhöhung dieser Wirtschaftsaktivitäten an sich jedoch nicht abwendbar sein. Eine gleichzeitige Reduktion ökologischer Auswirkungen erfordert ein hohes Maß an Innovation und Umdenken und ist im gegenwärtigen Ökonomie-Modell schwer realisierbar. Eine Veränderung der momentanen neo-klassischen Industrie- und Wirtschaftsmodelle ist daher dringend notwendig, denn diese wurden zu einem Zeitpunkt entwickelt, zu dem es auf der Welt noch viel weniger Menschen gab, einer sogenannten "leeren Welt" [5], in der sich die verwendeten Ressourcen in Relation zur Weltbevölkerung leicht wieder erholten und somit der vom Menschen erzeugte Einfluss von der Natur noch einigermaBen absorbierbar war. Heute lebt die Menschheit in einer vollen Welt, aber benimmt sich noch immer so, als wäre sie "leer", als gäbe es kein Limit der Ressourcen. Auch wenn verständlicherweise alle Menschen in Wohlstand leben möchten, sind die dafür notwendigen Industrieaktivitäten bei jetzt schon knapp $8 \mathrm{Mrd}$. Menschen innerhalb der ökologischen Grenzen des Planeten nicht mehr möglich, denn diese sind erreicht [3]. Es bedarf also einer nachhaltigen Wirtschaftsentwicklung, die das ökologische System als deren Basis anerkennt (Abb. 1).

\section{Nachhaltigkeit und Verantwortung}

Die Wörter "Nachhaltigkeit" und "Verantwortung " sind derzeit in allen Kontexten zu finden und werden oft als gleichwertig dargestellt. Es gibt dafür viele Definitionsversuche, die jedoch im Kern alle ähnlich sind [6]. Die wissenschaftstheoretische Diskussion und Reflexion dieser Begrifflichkeiten steckt noch in ihren Kinderschuhen und ist dringend notwendig, um deren Schwammigkeit aufzulösen [7]. Laut Vogt ist der normative Gehalt von "Verantwortlichkeit" vollkommen unklar, da sich jeder „verantwortlich" fühlt. Erst nach Auflösung dieser Unschärfe wird es möglich sein, dass daraus konkrete Wissenschaft abgeleitet werden kann, die es dann auch erlaubt, konkrete Maßnahmen für das Gesellschaftsmodell zu formulieren. Die SDGs sind ein guter erster Schritt in diese Richtung, bedürfen jedoch ebenfalls noch einer starken Nachschärfung in ihren einzelnen Themenfeldern. So sind sie eine gute Leitlinie und geben eine Entwicklungsrichtung vor, aber wie sie umgesetzt werden sollen und welche Synergien und Trade-offs sich hierbei ergeben, kann nur durch einen intensiven Arbeitsprozess herausgearbeitet werden. Das erreichen einzelner SDG-Targets kann nicht in Isolation stattfinden, da die Ziele miteinander verbunden sind und einander beeinflussen. Es gilt also noch klarer zu definieren, wie eine nachhaltig gestaltete Gesellschaft auszusehen hat, denn nur daraus können konkrete Aktionen abgeleitet werden, um die SDGs zu erreichen, was sich jedoch im gegenwärtigen System als schwierig herausstellt. Es scheint jedenfalls nicht sinnvoll zu sein, abrupte Veränderungen herbeizuführen, aus denen Systemzusammenbrüche resultieren könnten, sondern die Themen systematisch zu bearbeiten.
Eine sehr oft verwendete, jedoch ebenso unklare Definition für Nachhaltigkeit ist die aus dem UN Dokument "Our Common Future", dem sogenannten Brundtlant Report 1987 [8]: „Sustainable development meets the needs of the present without compromising the ability of future generations to meet their own needs." Diese Definition hat jedenfalls dafür gesorgt, dass die Nachhaltigkeit auf die Agenda der zukünftigen industriellen und wirtschaftlichen Entwicklung gesetzt wurde. Es gibt viele Maßnahmen, die sich mit verschiedenen Aspekten der Nachhaltigkeit auseinandersetzen bzw. versuchen, diese umzusetzen. Zunehmend zeigt sich jedoch die Komplexität der Problemstellung, die immer mehr in den Vordergrund rückt. Als eines von vielen systematischen Komplexitätsproblemen sei erwähnt, dass lokale Nachhaltigkeitsprobleme sich zu globalen ausgedehnt haben [9]. Beispielhaft hierfür ist die lokale $\mathrm{CO}_{2-}$ Vermeidung, die momentan nicht das globale $\mathrm{CO}_{2}$-Problem zu lösen oder gar den impliziten Import von $\mathrm{CO}_{2}$ durch Produkte zu verhindern vermag. Lokale Nachhaltigkeitsprobleme sind selten nur auf regionaler Ebene zu lösen, da die Globalisierung regionale und globale Handlungsräume miteinander verzahnt hat, und Strategien zur Resilienz sind nötig, um lokale Ökosysteme zu schützen und zu erhalten. Weiters trug zur systemischen Komplexität bei, dass spezifische und offensichtliche Verursacher zu impliziten Verursachern wurden: So werden zum Beispiel Schadstoffe, die aus einem Rauchfang kommen, heute durch Filter abgefangen, sind jedoch weiterhin in diesen Filtern zu finden, welche mit der Zeit ihre Schadstoffe wieder frei geben, wenn sie lediglich entsorgt werden. Dies ruft auch eine gewisse Zeitverzögerung der Schadstoffabgabe hervor [9], was das generelle Verständnis der Situation verzerrt. Die Ursache des Schadstoffproblems wurde nicht gelöst, verändert wurde nur der Umgang mit den Schadstoffen. Des Weiteren werden implizite Konsumenten-Emissionen, wie zum Beispiel Emissionen, die durch den Betrieb von Infrastruktur für den Konsum (Einkaufszentrum) oder für die Bereitstellung und den Betrieb von Konsumgütern (Straßen, Tankstellen) oder Konsumgüter, die durch Benützung als Schadstoffe (Mikroplastik, quarzhältiger Feinstaub) anfallen, nur langsam zum Thema in der Umweltdebatte. Sie sind nun letztendlich durch die SDGs durch ein eigenes Ziel, SDG 12 Responsible Consumption and Production, systematisch erfasst worden.

Ein weiterer Komplexitätsfaktor in der Nachhaltigkeitsfrage ist die immer größer werdende Anzahl an mineralischen Rohstoffen, die sich in einzelnen Produkten befinden. In einem modernen IT-Produkt stecken mehr als 40 Rohstoffe, um die wachsende Menge an technologischen Komplexitäten abzudecken. Dieser Umstand macht es zunehmend schwierig, diese Produkte am Ende ihrer Lebensdauer zu recyceln. Derzeit scheint das Konzept „Design for Recycling" nicht vorrangig. Kluge Recyclingkonzepte sind jedoch in allen Belangen notwendig, und Produkte müssen so komponiert sein, dass es am Ende ihres Produktlebens technisch möglich und ökonomisch interessant ist, sie einer Wiederverwertung zuzuführen. Ein zurzeit vielversprechendes Konzept ist jenes der Kreislaufwirtschaft, doch der Weg dorthin führt nicht nur über eine Weiterentwicklung der jetzigen linearen Wirtschaftskonzepte, sondern muss 

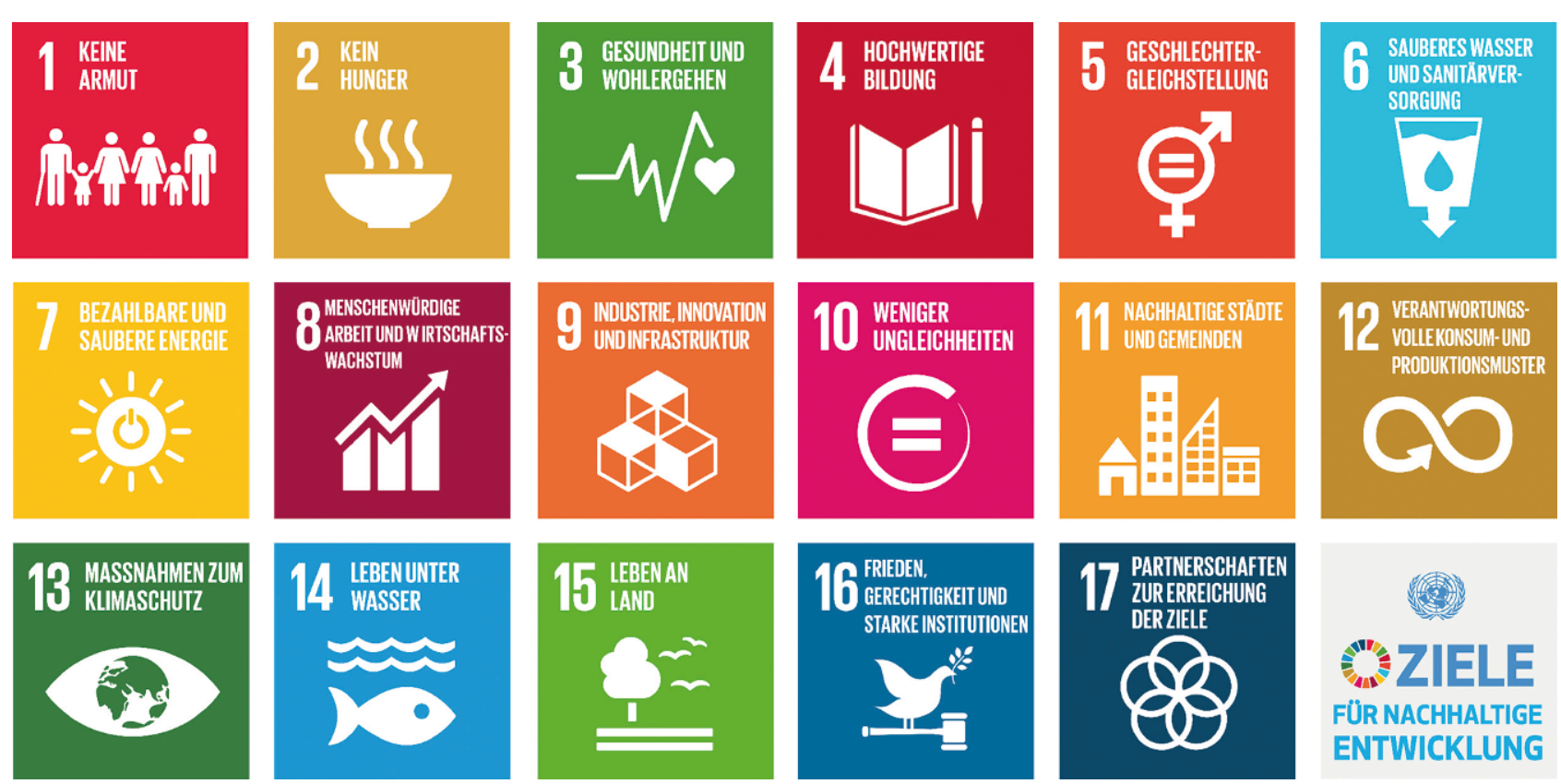

Abb. 1: 17 Sustainable Development Goals (www.un.org/sustainabledevelopment/news/communications-material/)

einer Transformation gleich kommen. Es braucht Veränderungen auf allen Ebenen, auf Produzenten-, Konsumentenund Recyclerebene sowie neue Wirtschaftsformen.

\section{Leistbare Rohstoffe in einem nachhaltigen System}

Neben der Herausforderung der systemischen Komplexität der Nachhaltigkeit ist eine weitere zukünftige und nicht minder komplexe Herausforderung die nachhaltige und leistbare Bereitstellung von primären mineralischen Rohstoffen, die durch den rasanten Anstieg des Bedarfs hervorgerufen wird, der gegenwärtig und auch in absehbarer Zukunft nicht durch Recycling abgedeckt werden kann. Es ist notwendig, hierfür einige Basisfaktoren heranzuziehen, die in Betracht genommen werden müssen, um dieses Thema erfolgreich zu entwickeln: Aspekte der Umwelt, des Sozialen, der Wirtschaft, der Bildung und Wissenschaft sowie der Politik. In einem starken Nachhaltigkeitsszenario, das also den Impakt der menschlichen Wirtschaftsaktivität für den Planeten wieder absorbierbar macht, ist es notwendig, die Umwelt als Basis von allem zu definieren und daraus dementsprechende planerische Schlüsse zu ziehen. Ein Bergbauprojekt muss demnach vom Beginn an so geplant werden, dass die Umweltimpaktfaktoren wie unter anderen $\mathrm{CO}_{2}$ Ausstoß, Wasserverbrauch, Einsatz von Chemikalien, Umgang mit Reststoffen oder mechanische Eingriffe in die Natur immer weiter zu minimieren sind und dass Umweltaspekte höchste Priorität haben [17]. Soziale Faktoren wie regionale Planung und Benefit Sharing, Arbeitssicherheit oder Schließungskonzepte müssen ebenfalls von Beginn an in eine Planung miteinbezogen werden. Wirtschaftliche Aspekte sind in Erwägung zu ziehen. So müssen lokal gesehen die Geschäftsmodelle die oben genannten Aspekte ermöglichen und auch weiterhin Gewinn abwerfen, um interessant zu bleiben, und global betrachtet die Rohstofflieferketten und der Rohstoffhandel transparenter gestaltet werden. Einhergehend damit ist eine permanente Notwendigkeit der interdisziplinären Beforschung dieser Themen, um einen solchen Fortschritt zu erlauben und voran zu treiben: Beispielsweise wird durch Erhöhung der technischen Effizienz der Ausbringung ein geringerer mechanischer Impakt erzeugt; oder die Interaktion von Gesellschaft und lokalen Projekten soll besser verstanden werden, um dementsprechende Regularien daraus entwickeln zu können; wie auch die Komplexität von Rohstofflieferketten weiter zu verstehen zu lernen ist, um daraus neue Aktionen wie Zertifizierung von Rohstoffen abzuleiten. Universitäten müssen diesem Thema größeren Raum geben und versuchen, mehr junge Leute auf Basis eines allgemeinen Grundlagenwissens zu Spezialisten auszubilden, die diese Fragenstellungen in Zukunft bearbeiten und lösen können. Ebenso sind Bildungsprogramme über die eigene Rolle in der Rohstofflandschaft für die breite Gesellschaft notwendig, um sie mehr in diese Themenstellungen zu integrieren und ihr so eine kompetente Stimme zu geben. Wie angedeutet, ist ein großer Teil eines solchen Unterfangens auch eine erfolgreiche nachhaltige Rohstoffpolitik, die sicherstellt, dass einerseits die beschriebene Entwicklung eine Selbstverständlichkeit ist und notwendige Regularien in Kraft sind und andererseits die Ressourcenbasis, in Form von sinnvollen Landnutzungskonzepten für zukünftige Generationen sichergestellt sind. Weiters muss so eine Politik gewährleisten, dass die Entwicklung von Rohstoffprojekten nicht primär der Spekulations-getriebenen Angebots- und Nachfragedynamik unterliegt, die eine hohe Preisvolatilität der Rohstoffpreise zur Folge hat [10] und oben genannte Parameter in Gefahr bringt. 
In allen angeführten Aspekten ist die Digitalisierung als Facilitator zur Zielerreichung nicht zu vernachlässigen, man beachte als positive Beispiele Hologramme von Bergbaubetrieben zur erleichterten Planung einer umweltfreundlichen, technisch und Ressourcen-effizienten Ausbringung, Virtual Reality in der Lehre zur Kompetenzsteigerung der zukünftigen Spezialisten oder verbesserte Überwachungsund Maintenance Systeme, die Unfälle vermeiden helfen.

\section{Industrielle Herausforderung und internationale Initiativen}

Aufgrund der Zunahme des Rohstoffverbrauchs ist es von großer Bedeutung, dass Industrien und Stakeholder, die mit der Bereitstellung und Verarbeitung von mineralischen Rohstoffen befasst sind - Bergbau, Aufbereitung und Metallurgie - sich trotz aller Schwammigkeit des Themas mit ökologisch und sozial verantwortlicher Produktion auseinandersetzen. In den letzten Jahren zeigen sich dazu einige positive Ansätze. Auf politischer Ebene hat die European Innovation Partnership on Raw Materials, die der Europäischen Kommission den politischen Weg zeichnet, 2017 in einem Positionspapier Ziele definiert, die das Meistern der drei Hauptherausforderungen der Industrie - Digitalisierung, Nachhaltigkeit und Innovation - fördern sollen, um den Übergang zu einer Niedrig- $\mathrm{CO}_{2}$-Kreislaufwirtschaft zu ermöglichen und gleichzeitig die Wettbewerbsfähigkeit zu erhalten [11]. Dabei setzen sie auf traditionelle marktregulierende Mechanismen und gehen mit den zu implementierenden Strategien davon aus, dass sich dadurch die Nachfrage nach nachhaltigen Produkten und einem zirkulären Konsum erhöhen wird.

Viele nicht politische Organisationen beschäftigen sich entweder auf wirtschaftlicher, ökologischer, sozialer oder rechtlicher Basis ebenfalls mit der verantwortlichen Förderung und Weiterverarbeitung von Rohstoffen. Unter vielen anderen sind erwähnenswerte Beispiele das International Council on Mining and Metals (ICMM), ein internationaler Zusammenschluss von 27 Bergbau- und MetallurgieFirmen, sowie 30 nationalen und regionalen Verbänden, bei dessen Arbeit die Stärkung der sozialen und ökologischen Aspekte der Bergbau- und Metallurgie-Industrie im Fokus steht [12]. Des Weiteren hat die Extractive Industries Transparency Initiative (EITI) [13] einen globalen Standard zum Management von Öl, Gas und mineralischen Ressourcen implementiert, der die derzeit 51 daran teilnehmenden Länder dazu verpflichtet, nach einem Standard zu berichten, der offenlegt, wer im Sektor unter welchen Voraussetzungen tätig ist, wo die Geldflüsse verlaufen und wem sie zugute kommen. Jeweils eine Koalition aus zuständiger Regierung, Firmen und Zivilgesellschaft unterstützt die Implementierung [12].

Die Organization for Economic Co-operation and Development (OECD) hat 2016 die bereits dritte Edition der Due Diligence Guidance for Responsible Supply Chains of Minerals from Conflict-Affected and High Risk Areas herausgegeben, die international als führender Standard in vielen Deklarationen als Referenz verwendet wird und Firmen dabei helfen soll, Menschenrechtsverletzungen zu vermeiden und die Versorgungsketten transparent zu halten. Die OECD hat viele weitere spezifische Dokumente zu dem Thema Extraktion und soziale Aspekte herausgegeben [14].

Das World Economic Forum [15] hat sich in den letzten Jahren ebenfalls verstärkt mit Extractive Industries auseinandergesetzt und sich durch Initiativen wie die Responsible Minerals Development Initiative 2013, Mining\&Metals in a Sustainable World 2015 und das Mapping Mining to the Sustainable Development Goals 2016 bemerkbar gemacht.

Der United Nations Environmental Programme UNEP International Resource Panel ist ein Zusammenschluss von Wissenschaftlern, gegründet im Jahr 2007, der unabhängige wissenschaftliche Bewertungen von politischen Reglementierungen bezüglich der nachhaltigen Nutzung von Naturressourcen erstellt, spezifisch für deren Umweltauswirkungen über einen Lebenszyklus betrachtet, um somit ein besseres Verständnis herzustellen, wie ökonomisches Wachstum von Umweltauswirkungen entkoppelt werden kann. Der Resource Panel veröffentlicht regelmäBig Reports über Resource Policy, Governance und Use. [16].

\section{Initiativen an der Montanuniversität}

Die Montanuniversität Leoben hat in den letzten Jahren ebenfalls viele Initiativen gesetzt, um die Rohstoffaktivitäten der Universität in Richtung Nachhaltigkeit zu lenken. All diese Initiativen sind im 2015 gegründeten Resources Innovation Center - RIC - ansässig und in permanenter Entwicklung, wie unter anderen die Beteiligung am EIT Raw Materials, EIT Climate-KIC und dem Projekt UniNetz, das die Implementierung der SDGs in Österreich voran bringen soll [18]. Das RIC, das der Nachhaltigkeit an der Montanuniversität selbst sowie deren Aktivitäten als Plattform nach außen hin nun ein Gesicht verleiht, beschäftigt sich schwerpunktmäßig mit interdisziplinären wissenschaftlichen Arbeiten über Rohstoffzyklen im Kontext der Nachhaltigkeitstheorien und Internalisierung von Externalitäten in Bezug auf Umweltbelastungen im Bereich Rohstoffproduktion und Rohstoffverarbeitung.

Eine interdisziplinäre Forschungsgruppe, die gemeinsam mit der TU Bergakademie Freiberg ins Leben gerufen wurde, beschäftigt sich mit sozialen Aspekten des Bergbaus. Masterarbeiten zur Auslotung von größeren Themengebieten, aus denen in weiterer Folge Projekte entstehen sollen, werden momentan ebenfalls erarbeitet, wie zum Beispiel die Nutzung von Rückständen aus der Rohstoffgewinnung, im konkreten Fall Feinschlämme aus der Flotation, zur nachhaltigen Fertigung von Betonfertigteilen insbesondere im Infrastrukturbereich oder auch die Behandlung des Themas Konfliktminerale und verantwortungsvolle Lieferketten von Rohstoffen. Gemeinsam mit dem MIRO, dem Montanuniversität International Relations Office, wurde 2018 erstmals eine Summer School zum Thema Circular Economy ins Leben gerufen, die durch ihre Konzeption intern interdisziplinär abgehalten wird, indem alle beteiligten Lehrstühle ihre Position im Rohstoff- und Materialkreislauf in Kontext setzen und den Studierenden so die Zirkularitätskonzepte der up- and downstream Inter- 
aktion und deren Interdependenz näher bringen. Parallel ist eine weitere Summer School, die sich 2019 mit dem Thema Ressourceneffizienz beschäftigt, mit externen österreichischen Nachhaltigkeitsstakeholdern und Partnern in Planung. International verstärkt wird das Thema im wissenschaftlichen Bereich durch eine Kooperation mit der IIASA - dem International Institute for Applied Systems Analysis in Laxenburg (http://www.iiasa.ac.at/).

Die verstärkte Kooperation der Montanuniversität Leoben mit der IIASA kommt zukünftig auch den Studierenden zugute, insbesondere im Rahmen der neuen Studienrichtung "Global Resources, Earth and Technology", einem Internationalen Masterstudium mit der Colorado School of Mines, das sich schwerpunktmäßig der nachhaltigen Nutzung von mineralischen Rohstoffen widmet.

Mit dem im Herbst 2017 verabschiedeten Entwicklungsplan hat die Montanuniversität Leoben ihre Position als führendes Mitglied der nationalen und internationalen Scientific Community im Bereich der Circular Economy wesentlich verstärkt. Sie ist in ihrer Profilierung mit ihren Fachgebieten gut sichtbar in den zukünftigen großen Themen positioniert und verfügt über eine beinahe geschlossene Wissensbasis, was eine der Grundvoraussetzungen für die qualitativ hochwertige Bewältigung der globalen Herausforderungen im Sinne der von den Vereinten Nationen formulierten Sustainable Development Goals ist. Mit der Mitwirkung an den großen globalen Forschungs- und Bildungsnetzwerken im Ressourcen- und Klimabereich und dem Forcieren von entsprechenden Lehr- und Forschungsaktivitäten finden sich die Visionen der Circular Economy auch verstärkt in den operativen Bereichen.

Funding. Open access funding provided by Montanuniversität Leoben.

Open Access Dieser Artikel wird unter der Creative Commons Namensnennung 4.0 International Lizenz (http://creativecommons.org/licenses/ by/4.0/deed.de) veröffentlicht, welche die Nutzung, Vervielfältigung, Bearbeitung, Verbreitung und Wiedergabe in jeglichem Medium und Format erlaubt, sofern Sie den/die ursprünglichen Autor(en) und die Quelle ordnungsgemäß nennen, einen Link zur Creative Commons Lizenz beifügen und angeben, ob Änderungen vorgenommen wurden.

Hinweis des Verlags. Der Verlag bleibt in Hinblick auf geografische Zuordnungen und Gebietsbezeichnungen in veröffentlichten Karten und Institutsadressen neutral.

\section{Literatur}

1. United Nations, World Population Prospects: The 2017 Revision: https://www.un.org/development/desa/publications/worldpopulation-prospects-the-2017-revision.html (10.01.2019)

2. Moser, P.; Tost, M.; Feiel, S.: Today's global overall resources consumption and the development since 1990, Vortrag Alpbach Technology Symposium Breakout Session 12: The Renewability of Societal Resources (24.08.2018)

3. Steffen, W.; Richardson, K.; Rockstrom, J.; Cornell, S.E.; Fetzer, I.; Bennett, E.M.; Biggs, R.; Carpenter, S.R.; De Vries, W.; De Wit, C.A.; Folke, C.; Gerten, D.; Heinke, J.; Mace, G.M.; Persson, L.M.; Ramanathan, V.; Reyers, B.; Sorlin, S.: Planetary boundaries: guiding human development on a changing planet. Science 347 (2015), issue 6223, 1259855

4. Steffen, W.; Broadgate, W.; Deutsch, L.; Gaffney, O.; Ludwig, C.: The trajectory of the Anthropocene: The Great Acceleration, The Anthropocene review, 2 (2015), issue 1, pp 81-98

5. Daly, H.: Great Transition Initiative, Economics for a full world, https://www.greattransition.org/publication/economics-for-a-fullworld (10.01.2019)

6. Industrie- und Handelskammer Nürnberg für Mittelfranken, IHK: Lexikon der Nachhaltigkeit, Begriffsentwicklung: https://www. nachhaltigkeit.info/artikel/begriffsentwicklung_1729.htm (20.01.2019)

7. Vogt, M.: Ethik der Wissenschaft in Zeiten des Klimawandels, Keynote-Vortrag und Diskussion, UniNEtZ Kick-off Veranstaltung, Wien (21.01.2019)

8. World Commission on Environment and Development-WCED: Our common future, Chapter 2: http://www.un-documents.net/ocf02.htm\#I (10.01.2019)

9. Holmberg, J.: Socio-Ecological Principles and Indicators for Sustainability. Chalmers University of Technology and Göteborg University, Institute of Physical Resource Theory, Diss., 1995

10. Moser, P.: Mineralische Rohstoffe: die Basis für Innovation und Nachhaltigkeit - Zur Zukunft des Bergbaus; in: Androsch, H.; Pelinka, P. (Hrsg.): Zukunft, Perspektiven einer neuen Welt; 1. Aufl., Wien: Springer, 2018, S. 249-255

11. EIP High Level Steering Group, European Innovation Partnership on Raw Materials: Position Paper on future Orientations (2017)

12. International Council on Mining and Metals: Our organization, 2019, https://www.icmm.com/ (23.01.2019)

13. Extractive Industries Transparency Initiative, Factsheet 2018: https:// eiti.org/document/eiti-fact-sheet (23.01.2019)

14. Organization for Economic Co-operation and Development, Due Diligence Guidance for Responsible Supply Chains of Minerals from Conflict-Affected and High-Risk Areas: http://www.oecd.org/ corporate/mne/mining.htm (23.01.2019)

15. World Economic Forum: Mining and Metals, 2019, https://toplink. weforum.org/knowledge/insight/a1Gb0000000pTDeEAM/explore/ dimension/a1Gb000000150oYEAU/publications (23.01.2019)

16. UNEP 2017: Reports and Data, 2018, http://www.resourcepanel.org/ (31.01.2019)

17. Tost, M.; Hitch, M.; Chandurkar, V.; Moser, P.; Feiel, S.: The state of environmental sustainability considerations in mining, Journal of cleaner production, 182 (2018), S. 969-977

18. Resources Innovation Center Leoben: Fostering Innovation Communities in the Raw Materials Field, 2018, www.ric-leoben.at (23.01.2019) 